\title{
Some problems in varieties of groups
}

\section{Warren Brisley}

This thesis is mainly concerned with metabelian p-groups; varieties of these are examined in the cases

(i) nilpotency class $<p$,

(ii) nilpotency class $p, p+1$.

In each case, the basis laws are determined for each variety of finite exponent, and a single generator found for each variety. For the small-class case, some results on critical groups are obtained; in particular, it is shown that each of the varieties can be generated by a critical group on two generators, and the proper subvariety of its proper factors is determined. Ascending chains of subvarieties are found from the proper factor variety to the original variety generated by the critical group. Various conjectures about critical groups are settled by examples drawn from the lattice of varieties of metabelian p-groups of small class.

Some results are obtained on regular metabelian p-groups of finite exponent, and a bound is given for the nilpotency class, independent of the number of generators of the group. Various consequences are found for varieties which can be generated by such groups, and some work of C.R. Hobby is extended to a variant of a theorem on "nearly-regular". p-groups, replacing "nearly-regular" by "metabelian, with a central element $z$ of order $p$ such that $G /\langle z\rangle$ is regular". Some recent work by P.M. Weichsel is examined from the results obtained, and some new proofs offered.

The following papers have arisen in part from the research for the the thesis:

Received 17 April 1969. Thesis submitted to the University of Newcastle, November 1968. Degree approved, March 1969. Supervisor: Dr I.D. Macdonald. 
Warren Brisley, "On varieties of metabelian p-groups and their laws", J. Austral. Math. Soc. 7 (1967), 64-80.

Warren Brisley, "Varieties of metabelian p-groups of class $p, p+1$ ", submitted to $J$. Austral. Math. Soc.

Warren Brisley and I.D. Macdonald, "Two classes of metabelian p-groups", Math. 2. 112 (1969), 5-12. 